
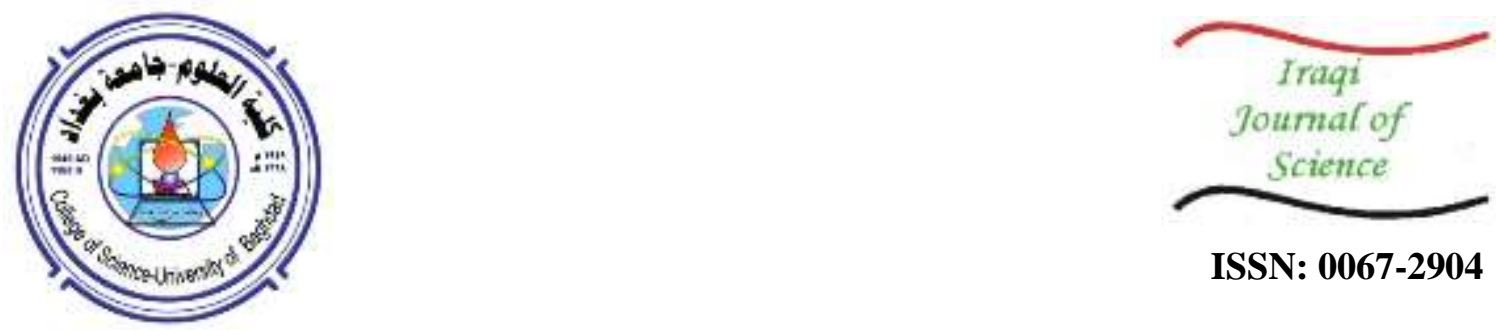

ISSN: 0067-2904

\title{
A Study on the Scattering and Absorption Efficiencies of Si-Ag Coaxial Nanowire
}

\author{
Jamal M. Rzaij*, Sameer O. Nawaf, Abdul Salam Mohammed Khalaf \\ Department of Physics, College of Science, University of Anbar, Ramadi, Iraq
}

\begin{abstract}
Scattering and Absorption Efficiencies of Si-Ag Coaxial nanowire (NWs) were simulated using Mie-Lorentz scattering approach. The thickness of Ag shell was fixed at around $10 \mathrm{~nm}$ with Si core diameter of (10, 20, 30 and 40) nm. Scattering Efficiencies $\left(Q_{s c a}\right)$ and Absorption Efficiencies $\left(Q_{a b s}\right)$ of core-shell nanowire as a function of wavelength $(300-2000 \mathrm{~nm})$ within various core diameters were calculated. The study shows a remarkable behavior of scattering for un-polarized light in Silicon nanowire (core only) with wavelength of (320-500nm). In other words, adding $\mathrm{Ag}$ shell has decreased the scattering efficiency of core-shell nanowire for all diameters.
\end{abstract}

Keywords: Mie scattering, scattering efficiency, Absorption efficiency, Si-Ag Coaxial nanowires.

\section{دراسة كفاءة الاستطارة والامتصاص لأسلاك Si-Ag المحورية النانوية}

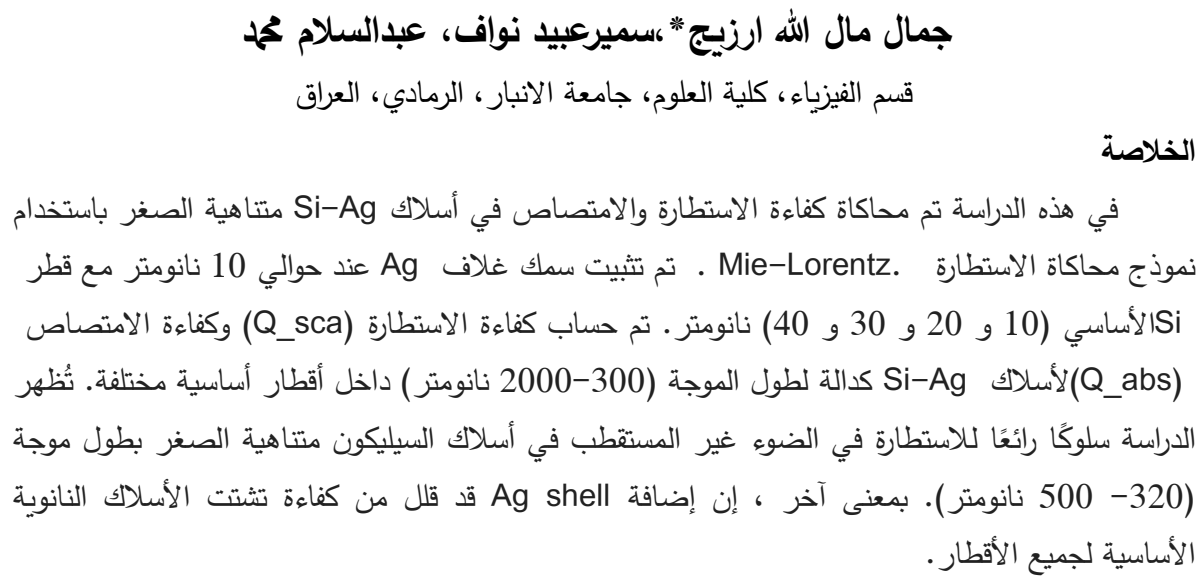

\section{Introduction}

A Single nanowire is a nanostructure with a diameter of a nanometer [1]. Silicon nanowire, also denoted to as SiNW, is a type of nanowire mainly formed from a silicon precursor by the engraving of a solid or through catalyzed growth from a vapor or liquid phase. As a result of its unique structural, optical, and electrical properties that are not seen in bulk Silicon materials, Si-NW occupies a special position as a subject of numerous researches and applications[2-5]. Silicon nanowires Si-NWs are a main research subject in the field of nanotechnology owing to their uniquely physical and chemical properties such as high selective adsorption of gas, novel thermoelectricity, and enhanced biological and chemical sensitivity[6]. Optical properties act an important role in investigates of the synthesis of

*Email: jam72al@gmail.com 
semiconductor materials. In order to study the photonic applications of Si-Ag coaxial NWs Scattering and Absorption Efficiencies of Si-Ag NWs were calculated.

\section{Theoretical method:}

The optical properties and photocurrent density of Si-Ag core-shell nanowires have investigated using Mie- Lorenz scattering theory. The simulation tools are available at nanoHUB website [7]. It was improved to evaluate the circular cylinders with both core and core-shells forms. The calculation of light absorption of single coaxial nanowires sourced the simulator "Optical Properties of Single Coaxial Nanowires"[7]. As presented in Figure-1, the coaxial nanowires were evaluated as limitless long cylinders along the z-axis, typically lighted by an electromagnetic plane wave within an incident transmission wave vectork. The radii of coaxial nanowires are given by a and $b$, correspondingly. The angle of incidence transmission wave is symbolized by $\alpha$. The simulation considers that transverse electric (TE) wave and the transverse magnetic (TM) wave are both perpendicular to the axis of coaxial nanowire.
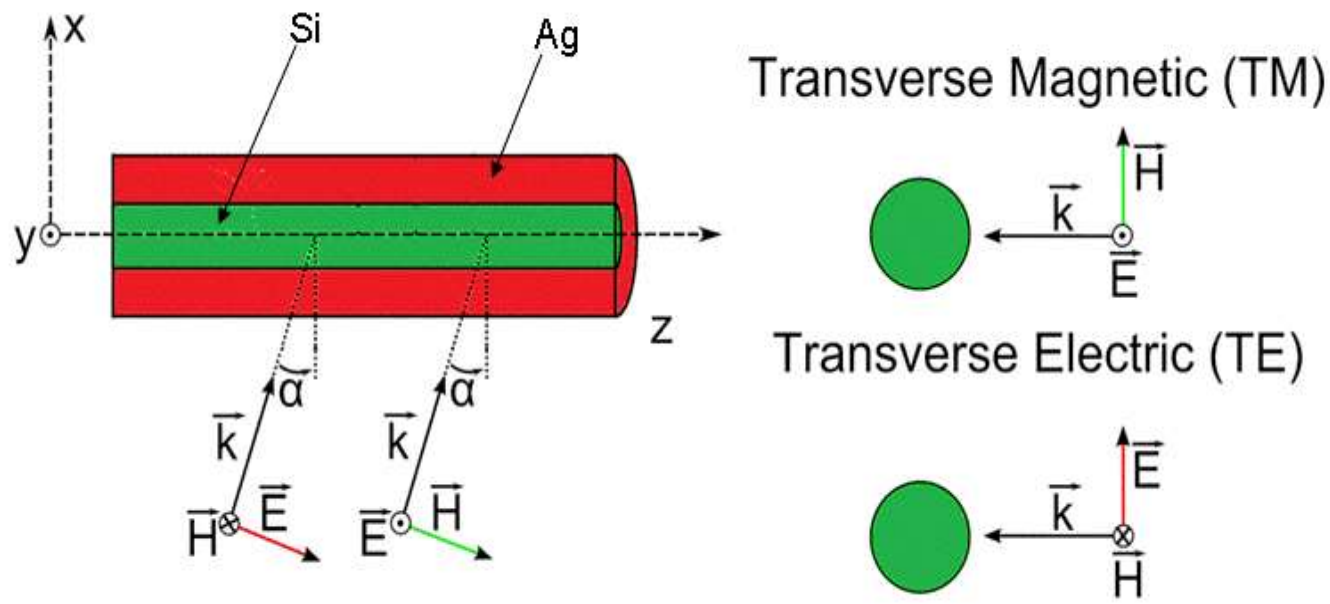

Figure 1-The Schematics of coaxial nanowire with the direction of TM and TE propagation.

The absorption efficiency $Q_{a b s}$ of a single wire is given by

$$
Q_{a b s}=C_{a b s} / C_{g e o m}
$$

where $C_{a b s}$ and $C_{g e o m}$ are the absorption cross-section and geometrical cross sections respectively [8].

The absorption cross section is written by

$$
C_{\text {abs }}=C_{\text {ext }}-C_{\text {scat }}
$$

Where $C_{\text {ext }}$ and $C_{\text {scat }}$ are the extinction cross section and scattering cross section respectively.

In case of un-polarized light, the absorption cross section $C_{a b s}$ of core-shell nanowires can be denoted as [9]:

$$
C_{a b s}=\left(C_{\text {ext }}^{T E}+C_{\text {ext }}^{T M}\right) / 2-\left(C_{\text {scat }}^{T E}+C_{\text {scat }}^{T M}\right) / 2
$$

The following procedure has been applied to estimate the Mie coefficients of the system:

1. Find out the solutions of the $2 \mathrm{D}$ vector wave equation in several spatial regions to examine the electromagnetic fields.

2. Substituting the magnetic field to the electric field of Maxwell's equations.

3. Writing these solutions in formula of a summation for Bessel functions whose coefficients were determined by resolving the boundary conditions.

4. The solutions are then denoted in a matrix form.

5. Calculating the unknown Mie coefficients.

\section{Results and discussion}

\subsection{The Scattering efficiency for un-polarized light}

Scattering from NWs is directly comparative with the scattering efficiency of NWs and illumination [10]. Figure- 2 shows the relation between $\left(Q_{s c a}\right)$, for un-polarized light, as a function of wavelength for various core diameters. In the case of Silicon nanowire (core only), a remarkable 
behavior of scattering was observed in the wavelength range 320 to $400 \mathrm{~nm}$ with a sharp peak at 400 $\mathrm{nm}$. This followed by a decrease in $\left(\mathrm{Q}_{\mathrm{sca}}\right)$ value with increased the wavelength to approaching zero along the studied range of spectrum [11]. For Si-Ag samples with different radius of Si core, there was a marked decrease in $\left(Q_{\text {sca }}\right)$ values with increasing core radius. In addition, a number of peaks observed at different position of wavelength 380, 480, 430, 420 and $450 \mathrm{~nm}$ for increasing wire diameter of the $\mathrm{Si}-\mathrm{Ag}$ coaxial nanowires to $10,20,30$ and $40 \mathrm{~nm}$, respectively. In the range of 840$2000 \mathrm{~nm}\left(\mathrm{Q}_{\mathrm{sca}}\right)$, slightly increased with increasing of $\mathrm{Si}$ core-Ag. This is because the scattering efficiency depends on the size of these nanowires [12]. Consequently, this information can be used in the evolution of the $\mathrm{Si}-\mathrm{Ag}$ coaxial nanowires.

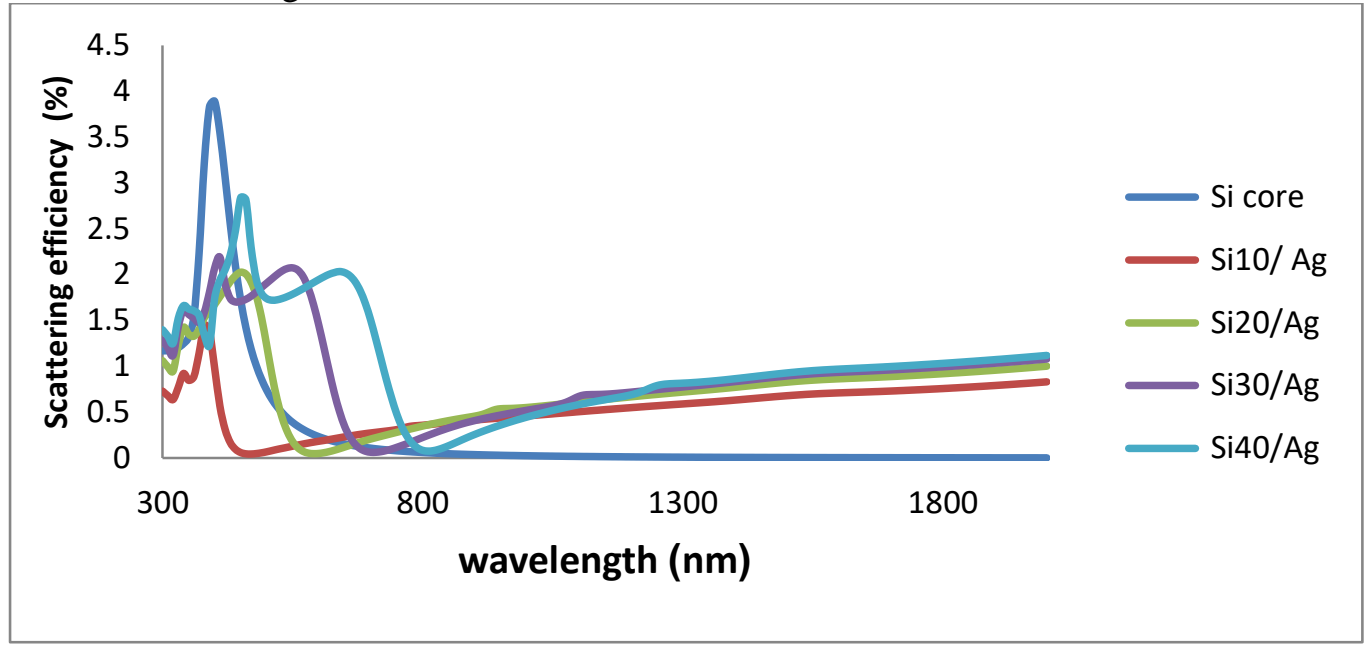

Figure 2-Scattering efficiency for un-polarized light as a function of wavelength for various core diameters.

\subsection{The Scattering efficiency for (TE)}

Figure-3 indicates the relation between the scattering efficiency for the transverse electric (TE) wave as a function of wavelength for various core diameters. It was clear that scattering efficiency of Silicon nanowire (core only) was close to zero along the range of spectrum under study. The reason for this is that the silicon refractive index, in this case, is equal even though the wavelength of the falling radiation has increased, so there was no scattering to the falling light wave within this spectral range [13]. The calculations indicated high-value $\left(Q_{\text {sca }}\right)$ within the visible spectrum and decreased significantly in the infrared region of the spectrum, where it upsurge with increasing nanowire diameter of shell coating ( $\mathrm{Si}-\mathrm{Ag}$ ) nanowires from 300 to $460 \mathrm{~nm}$. The scattering efficiency was approximately equal to zero at wavelengths 900 to $2000 \mathrm{~nm}$ for all Si-Ag diameters.

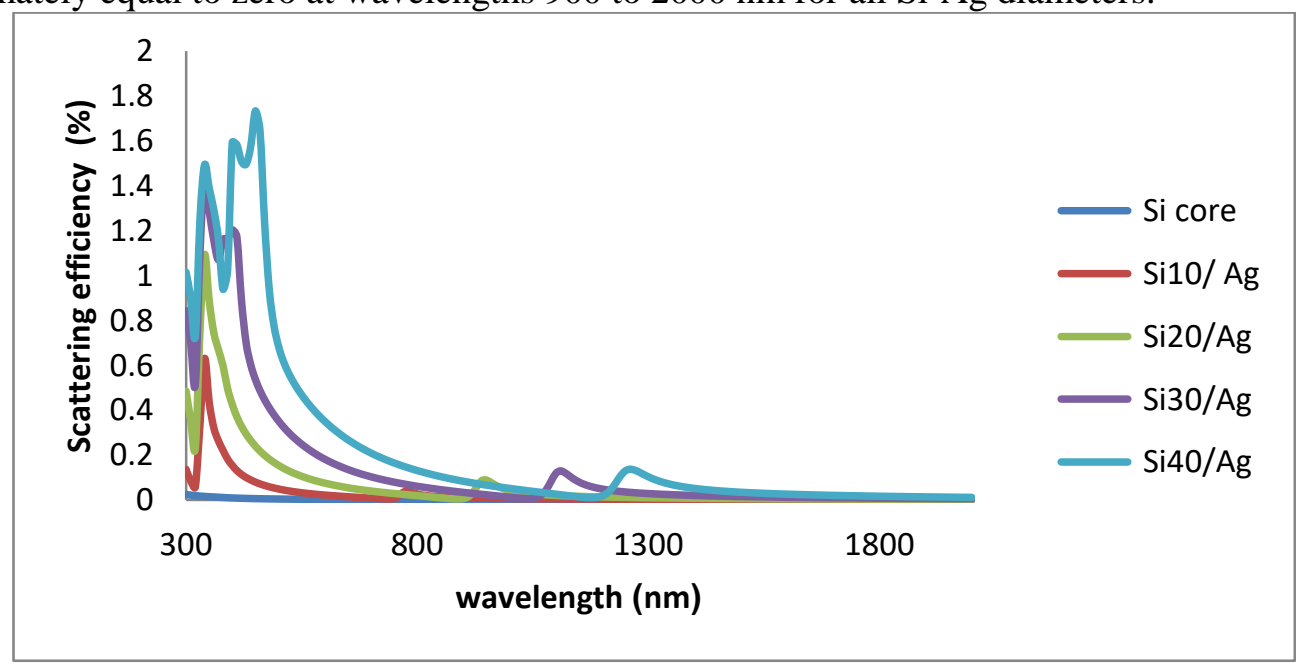

Figure 3-Scattering efficiency for the transverse electric (TE) wave as a function of wavelength for various core diameters. 


\subsection{The Scattering efficiency for (TM)}

Figure- 4 shows the relation between the scattering efficiency for the transverse magnetic (TM) wave as a function of wavelength for various core diameters. At wavelength of $390 \mathrm{~nm}$, there was a bulky scattering efficiency of (TM) wave for $\mathrm{Si}$ core. This behavior is comparable of the $\left(\mathrm{Q}_{\mathrm{sca}}\right)$ performance for un-polarized light at such wavelength. In case of $\mathrm{Si}-\mathrm{Ag}$ core-shell, scattering efficiency decrease and close to zero at 450, 570, 700 and $800 \mathrm{~nm}$ for (Si10-Ag, Si20-Ag, Si30-Ag, and $\mathrm{Si} 40-\mathrm{Ag}$ ) diameters respectively. This is due, again, to an increase in the size of the model ( $\mathrm{Si}-\mathrm{Ag}$ ) rapidly reduces the degree of polarization as a result of $\mathrm{Ag}$ shell presence [14] While there was a linear increase in the $\left(Q_{\text {sca }}\right)$ with increasing of wavelength.

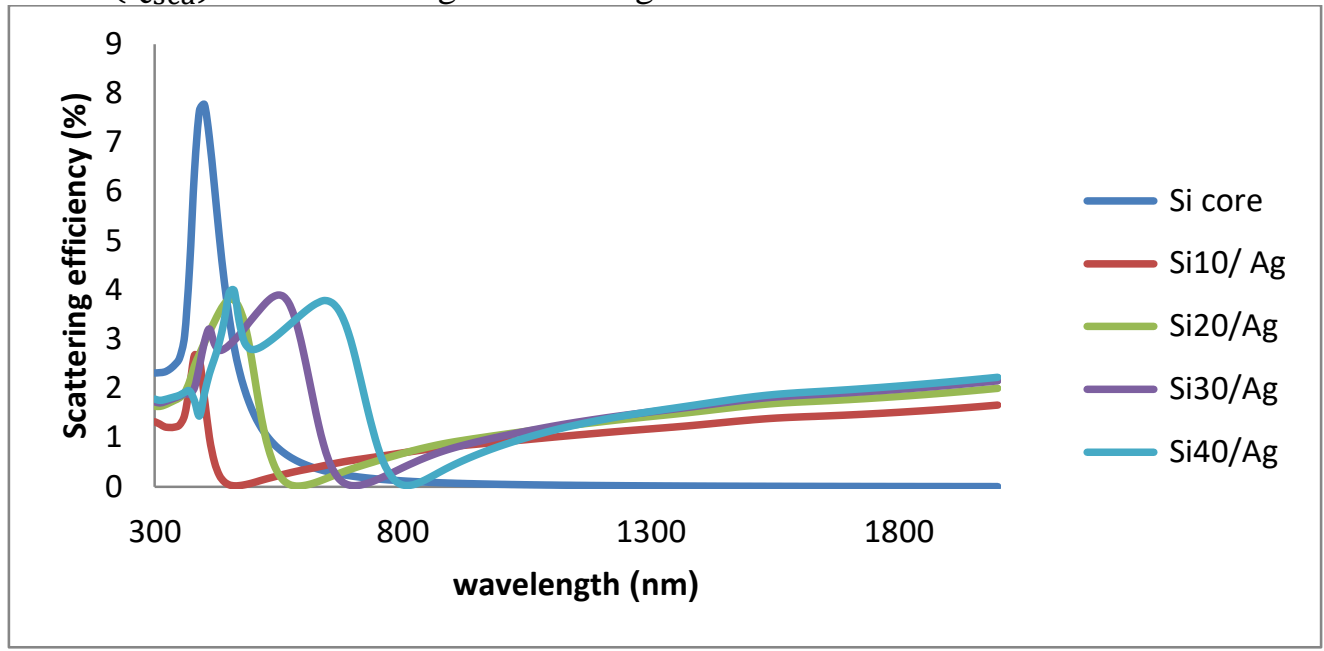

Figure 4-Scattering efficiency for the transverse electric (TM) wave as a function of wavelength for various core diameters.

\subsection{The absorption efficiency for un-polarized light}

Figure-5 shows $Q_{a b s}$ spectrum for un-polarized light within the range (300-2000) nm. It reveals a high $Q_{a b s}$ value within visible spectrum range, followed by a decreasing towards the range of the infrared spectrum. The differences in the $Q_{a b s}$ values due to the difference in the structure volumes, same results with [15]. For silicon nanowire (core only), $Q_{a b s}$ approaching zero within IR-spectrum, while there was a slight linear increase with increased wavelength accompanied by an appearance of number peaks at 770, 930, 1100 and $1230 \mathrm{~nm}$ depending on increasing of core radii- shell (Si-Ag). The reason for the presence of these peaks is due to the increase in the number of modes within the cylinder model of nanowires [15]. This finding confirms that the radii of nanowires have a significant effect on the absorption spectrum where absorption increases with increased volumes of nanowires.

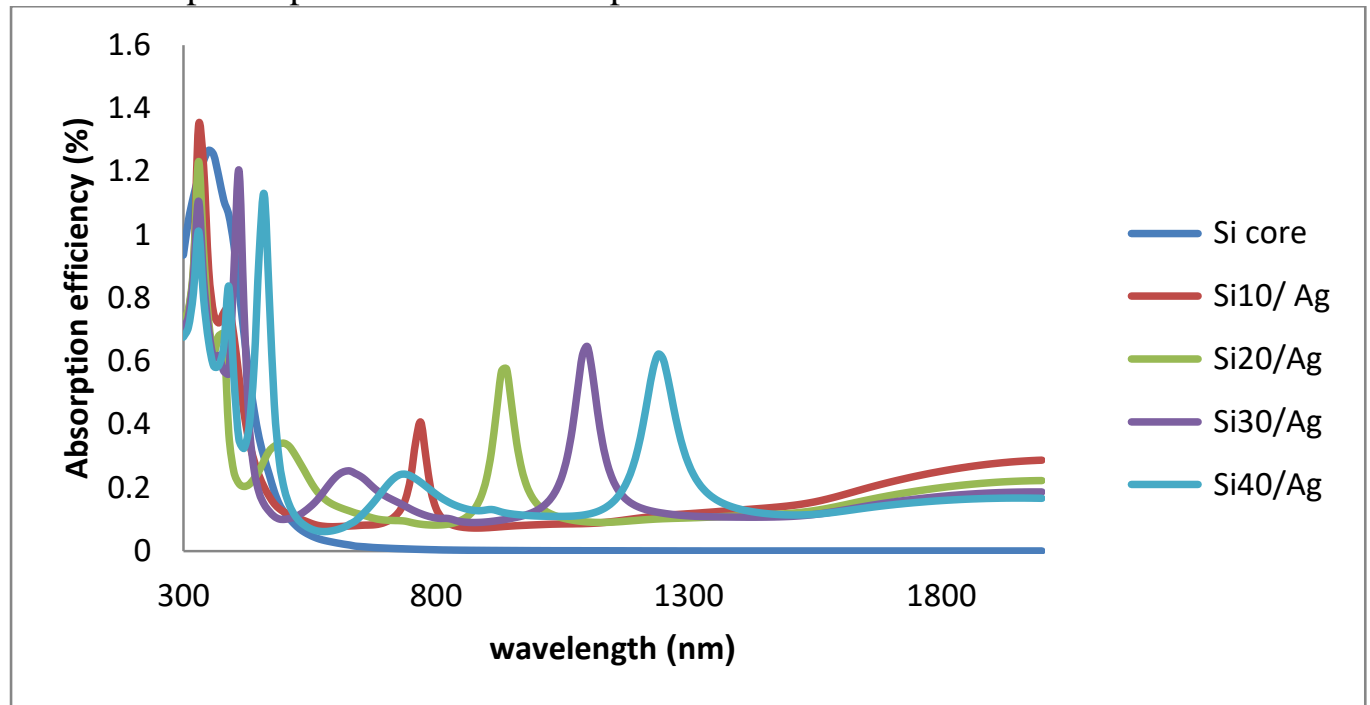

Figure 5-Absorption efficiency for un-polarized light as a function of wavelength for various core diameters. 


\subsection{The absorption efficiency for (TE)}

Figure-6 demonstrates the $Q_{a b s}$ for (TE) wave as a function of wavelength for various core diameters. A remarkable behavior with absorption efficiency for (TE) close to zero was observed for Silicon nanowire (core only) for all wavelengths from $300 \mathrm{~nm}$ to $2000 \mathrm{~nm}$. The reason for this is that the silicon refractive index, in this case, is equal even though the wavelength of the falling radiation has increased. An equivalent sharp peak of $Q_{a b s}$ for all diameters at with wavelengths $(300-360 \mathrm{~nm})$ have been noticed at the case of $\mathrm{Si} / \mathrm{Ag}$ core-shell. Also, $\mathrm{Q}_{\mathrm{abs}}$ has clear peaks at wavelengths (770, 930, 1100, $1240 \mathrm{~nm}$ ) for (Si10-Ag, Si20-Ag, Si30-Ag, Si40-Ag) diameters). This behavior is comparable of the absorption efficiency $Q_{a b s}$ performance for un-polarized light at such wavelength.

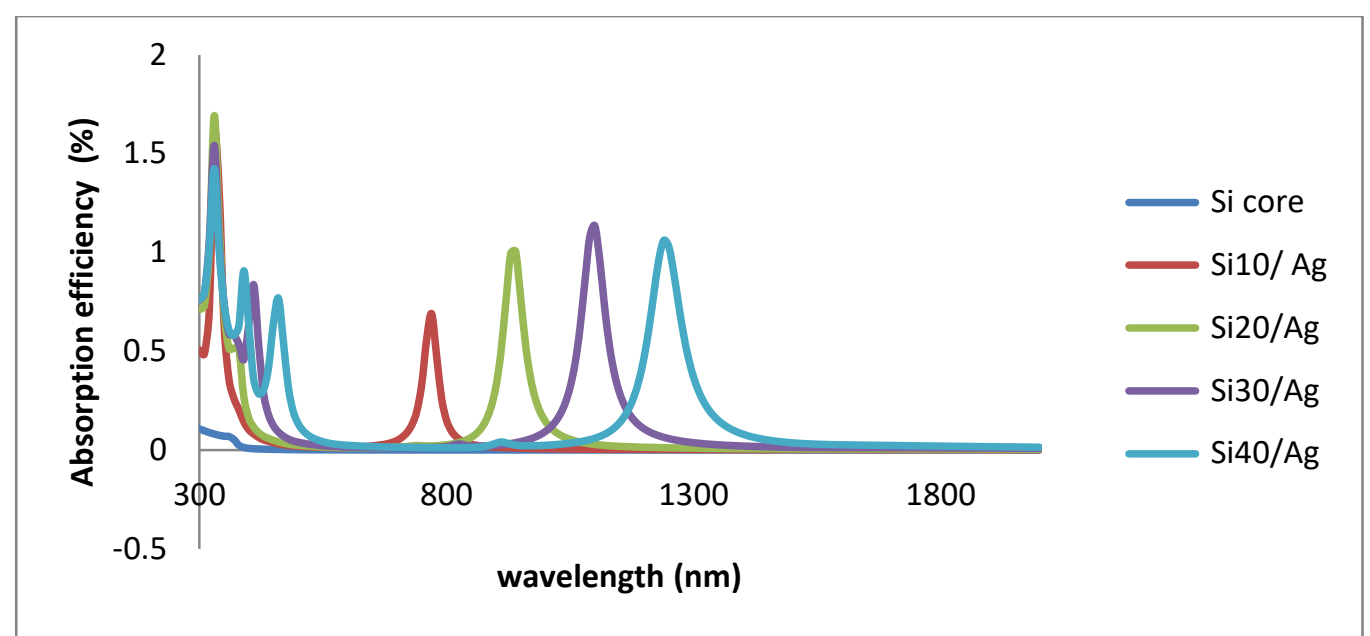

Figure 6-Absorption efficiency for the transverse electric (TE) wave as a function of wavelength for various core diameters.

\subsection{The absorption efficiency for (TM)}

Figure-7 shows the relation between the $Q_{a b s}$ for (TM) wave as a function of wavelength for various core diameters. For Si core, it was noticed a sharp peak at range of UV spectrum and it was close to the results obtained by Syed Hamad [16]. At the wavelengths between (900- 1400) nm, it was seen the absorption efficiency $Q_{a b s}$ values are degenerate for all nanowire diameters due to the approximately identical real parts of the complex refractive indices of $\mathrm{Si}[17,18]$.

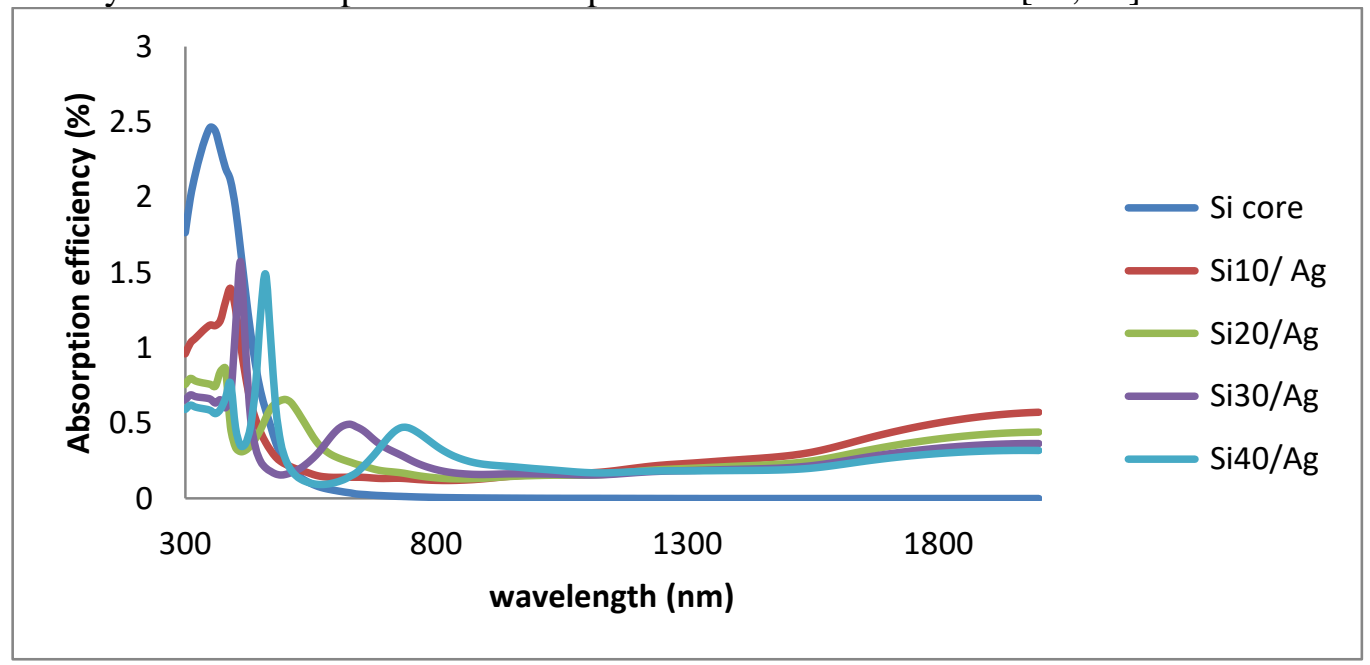

Figure 7-Absorption efficiency for the transverse electric (TM) wave as a function of wavelength for various core diameters 


\section{Conclusion:}

In summary, it was verified the scattering and absorption efficiencies of Si-Ag Coaxial nanowire by theoretical online simulation using the Mie scattering method. It was shown that adding Ag shell has decreased the scattering efficiency of core-shell nanowire for all diameters. Scattering efficiency of (TM) wave was equivalent to scattering efficiency for un-polarized light at exact wavelengths. Likewise, Absorption efficiency of (TE) wave was equivalent to absorption efficiency for un-polarized light at exact wavelengths. From the discussion of scattering and absorption for coaxial $\mathrm{Si}-\mathrm{Ag}$ nanowires, it is clear that nanowires can play an extremely important role in the development of optoelectronic devices.

\section{References}

1. Morral, A.F.I. 2011. Gold-free GaAs nanowire synthesis and optical properties, IEEE J. Sel. Top. Quantum Electron., 17(4): 819-828,.

2. Liu, M., Jin, P., Xu, Z., Hanaor, D.A.H., Gan, Y. and Chen, C.Q. 2016.Two-dimensional modeling of the self-limiting oxidation in silicon and tungsten nanowires, Theor. Appl. Mech. Lett., 6(5): 195-199,.

3. Peng, K.Q., Wang, X., Li, L., Hu, Y. and Lee, S.T. 2013. Silicon nanowires for advanced energy conversion and storage, Nano Today, 8(1), :75-97.

4. Cui, Y. and Lieber, C.M. 2001. Functional nanoscale electronic devices assembled using silicon nanowire building blocks, Science (80-. )., 291(5505): 851-853.

5. Ibrahim I.M., Rzaij J.M. and Ramizy A. 2017. Characterization of CuPcTs/Ps for No2 gas sensor. Dig. J. Nanomater. Biostructures. 12(4):1187-1196

6. Wu, S.L., Zhang, T., Zheng, R.T. and Cheng, G.A. 2012. Photoelectrochemical responses of silicon nanowire arrays for light detection, Chem. Phys. Lett., 538: 102-107.

7. Sarath, R., Tzu-ging, L., Elizabeth, H.K. and Chen, Y. 2015. Optical Properties of Single Coaxial Nanowires.

8. Bohren, C.F. and Huffman, D.R. 1983. Absorption and scattering of light by small particles. Wiley.

9. Liu, W.F., Oh, J.I. and Shen, W.Z. 2011. Light trapping in single coaxial nanowires for photovoltaic applications. IEEE Electron Device Lett., 32(1): 45-47.

10. Misbah, I., Ahmed, S., Muyeed, A., Babuya, S.K. and Khabir, M. 2015. Study on the A Bsorption And Scattering, 4(4): 23-29.

11. Gu, X., Taylor, R., Dombrovsky, L., Timchenko, V. and Heng Yeoh, G. 2018 . The Effect of Gold Nanorods Clustering on Near-Infrared Radiation Absorption, Appl. Sci.,8 (7): 1132.

12. Ibraheam, A.S., Rzaij JM, Fakhri MA. and Abdulwahhab AW. 2019. Structural, optical and electrical investigations of $\mathrm{Al}: \mathrm{ZnO}$ nanostructures as UV photodetector synthesized by spray pyrolysis technique. Materials Research Express. 6 (5).

13. Haverkate, L.A. and Feiner, L.F. 2006. Optical properties of cylindrical nanowires. npublished Philips Research Technical Note, PR-TN 2006- (2006).

14. Horvath, H. 2009. Gustav Mie and the scattering and absorption of light by particles: Historic developments and basics," J. Quant. Spectrosc. Radiat. Transf., 110(11): 787-799.

15. Ali, L. 2017 . Investigation the Absorption Efficiency of GaAs / InGaAs Nanowire Solar Cells Highlights.

16. Hamad, S., Mechanics, F., Pathak, A.P., Tewari, S. and Soma, V. S. 2014 .Venu JPCC2 2014.

17. Liu, W.F., Oh, J.I. and Shen, W.Z. 2011 .Light absorption mechanism in single c-Si (core)/a-Si (shell) coaxial nanowires. Nanotechnology, 22(12).

18. Rzaij JM, Ibrahim IM, Alalousi MA, Habubi NF. 2018. Hydrogen sulfide sensor based on cupric oxide thin films. Optik.,1(172): 117-26. 